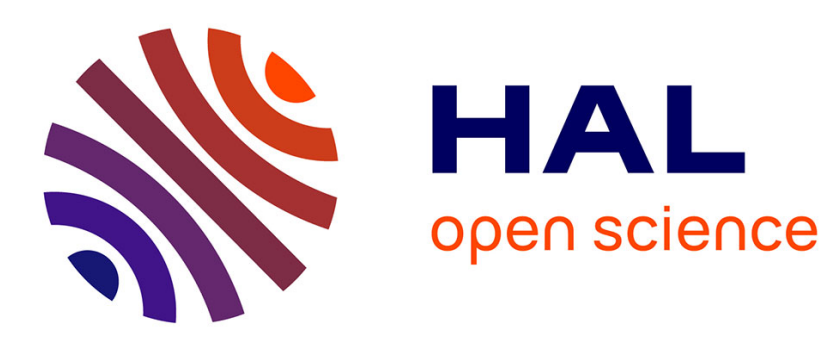

\title{
Le caché de La Poste La genèse de temps virtuels pour organiser le travail des facteurs
}

\author{
Nicolas Jounin
}

\section{To cite this version:}

Nicolas Jounin. Le caché de La Poste La genèse de temps virtuels pour organiser le travail des facteurs.

La Revue de l'IRES, 2018, 10.3917/rdli.093.0025 . hal-01857467

\section{HAL Id: hal-01857467 \\ https://hal.science/hal-01857467}

Submitted on 16 Aug 2018

HAL is a multi-disciplinary open access archive for the deposit and dissemination of scientific research documents, whether they are published or not. The documents may come from teaching and research institutions in France or abroad, or from public or private research centers.
L'archive ouverte pluridisciplinaire HAL, est destinée au dépôt et à la diffusion de documents scientifiques de niveau recherche, publiés ou non, émanant des établissements d'enseignement et de recherche français ou étrangers, des laboratoires publics ou privés. 


\title{
Le caché de La Poste \\ La genèse de temps virtuels pour organiser le travail des facteurs
}

\author{
Nicolas JOUNIN $^{1}$
}

La Poste détermine le circuit des tournées de ses facteurs en référence à la durée nécessaire au tri et à la distribution du courrier correspondant à ce circuit. Jusqu'au début des années 2000, elle le faisait au moyen du chronométrage des différentes étapes de chaque tournée. Depuis, elle a accéléré la révision des tournées en mettant en œuvre des combinaisons automatiques de l'estimation du trafic, du référencement de la géographie, et de vitesses et temps standards prédéterminés. Bien que ce dispositif ait été réfléchi depuis des décennies, il n'a été mis en œuvre qu'à la faveur de la réduction de la durée légale du travail et de la diminution du trafic de courrier. Aujourd'hui, il fait l'objet d'une controverse qui porte notamment sur les fondements des vitesses et temps standards conçus dans les années 1990. Leur validité est mise en cause par des facteurs et leurs représentants, et La Poste ne peut la défendre car elle affirme ne plus disposer des documents rendant compte de la conception de ses propres «normes et cadences».

Parmi les postiers, ceux qui sont connus sous le nom de «facteurs » travaillent dans des « plateformes de distribution du courrier ». On compte en France un peu moins de 3000 de ces plateformes, également nommées « centres courrier» ou simplement « bureaux ». Depuis celles-ci, les facteurs assurent « la logistique du dernier kilomètre », c'est-à-dire qu'ils accomplissent le dernier tri (après ceux opérés dans la trentaine de «plateformes industrielles du courrier ») et la distribution aux destinataires individuels de plis et d'objets. Les facteurs participent aussi et simultanément au travail de l'amont, c'est-à-dire la collecte du courrier, par exemple en

1. Sociologue, maître de conférences (en disponibilité) de l'université Paris 8 - Saint-Denis. L'auteur remercie Sébastien Chauvin, Brice Guillotin, Karim Hammou et Léo Reynes pour leurs lectures et commentaires de différentes versions de l'article. 
relevant le contenu des « boîtes jaunes ». Mais l'essentiel de leur temps de travail est constitué par le tri et surtout la distribution des objets destinés aux institutionnels et aux particuliers domiciliés sur un parcours déterminé, qui constitue ce qu'on appelle ordinairement la « tournée ». Chaque jour, le facteur est amené à arpenter l'ensemble de ce parcours pour remettre la totalité du courrier qu'il a réceptionné le matin même.

Depuis le milieu des années 2000, La Poste met en œuvre une révision régulière de ces tournées, centre par centre, qui débouche presque systématiquement sur la suppression d'une partie d'entre elles et le rallongement des tournées restantes. Ce mouvement a conduit à la disparition d'environ 2000 «bureaux », 15000 tournées (sur 70000 ) et 25000 emplois de facteurs sur 100000 (La Poste, 2002 ; Cour des comptes, 2016a). L'objectif est de contrer les effets de la baisse du volume d'objets échangés. L'accélération du rythme des « réorganisations » a été permise par l'usage de nouveaux outils de détermination du circuit des facteurs.

Comment est défini le périmètre d'une tournée de facteur? Ou, plutôt, à partir de quels fondements est-il périodiquement redessiné, raccourci ou (plus souvent) allongé ? Le parcours est décomposé en morceaux, ayant chacun leurs distances, leurs types d'usagers et de boites aux lettres, leur volume de lettres et colis. Ces éléments contribuent à déterminer la charge de travail sur les morceaux du parcours, une charge que La Poste exprime en durée. Une direction locale d'établissement a terminé de dessiner le parcours quand l'agrégation des morceaux dudit parcours atteint la durée convenue pour la tournée, elle-même dépendante de la durée légale du travail. La question devient donc : comment La Poste attribue-t-elle des durées aux morceaux de parcours?

Au milieu des années 2000, l'entreprise a discrètement transformé sa manière de faire. Pour comprendre ce changement de méthode, faisons une comparaison. Imaginons qu'un parent doive faire évaluer le poids de son enfant par le pédiatre. Première solution : le parent amène l'enfant chez le pédiatre, qui le pose sur sa balance et enregistre directement le poids mesuré. La balance est un instrument de mesurage, dont l'équivalent est pour les facteurs le chronomètre du "vérificateur », qui «accompagne » les tournées. C'est la méthode appliquée par La Poste jusqu'au début des années 2000. Bien sûr, il y a des biais possibles : la balance est mal réglée, ou bien l'enfant n'a pas exactement son poids habituel, car il sort d'un repas qui l'a alourdi ou d'une gastro-entérite qui l'a amaigri. Le pesage du jour n'est pas forcément représentatif. Seconde solution : le parent se rend chez le pédiatre sans son enfant. Il explique au médecin ce que son enfant a mangé, le contenu et la taille des petits pots, mais aussi ses activités quotidiennes, la fréquence et la nature de ses selles, etc. Le pédiatre note consciencieusement toutes ces informations et, au moyen de formules préétablies, en 
déduit le poids de l'enfant qu'il n'a pas vu. C'est cette seconde solution qui est aujourd'hui appliquée à La Poste pour évaluer la durée des tournées. Le pédiatre n'est plus tributaire des mauvais réglages de sa balance, ou de l'état particulier de l'enfant le jour de l'examen clinique. Mais cela ne veut pas dire que l'on a éliminé tous les biais (sinon les pédiatres ne verraient plus que des adultes seuls qui leur décrivent des enfants). Les biais possibles sont ailleurs : ils dépendent de la fiabilité des informations remontées par le parent, du nombre d'éléments de description de l'enfant que le modèle de calcul du pédiatre est susceptible d'intégrer, et pour finir du bien-fondé des formules qui calculent le poids.

Laissons l'exemple imaginaire de l'enfant et du pédiatre, et revenons aux tournées des facteurs : la première solution, celle du chronométrage, n'existe plus ; seule subsiste la seconde, c'est-à-dire le calcul de la durée de la tournée au moyen de formules inscrites dans un logiciel. Le mesurage a été délaissé au profit du calcul, présenté aux facteurs comme scientifique, objectif et indiscutable.

Ce calcul combine trois types de données : 1) une schématisation de l'environnement géographique et postal (par le recensement de distances, des boîtes aux lettres...) ;2) une estimation, pour chaque « point de distribution ", du volume de trafic (nombre moyen de lettres, de colis, de recommandés...) ; 3) des cadences conventionnelles, établies par le siège national de La Poste et s'étendant à l'ensemble du territoire. Si les deux premiers types de données font l'objet de procédés de collecte standardisés, ils restent soumis à des pratiques et des manipulations locales diversifiées. En revanche, les vitesses et temps standards constituent un puissant principe d'unification et d'homogénéisation imposé par le siège, à tel point que les directions locales sont conduites à les appliquer sans en connaitre les fondements.

Leur existence et leur usage correspondent à ce que Hatzfeld (2005) a nommé des "temps virtuels », ce que leurs fondateurs dans l'industrie avaient baptisé « temps standards », et ce que La Poste appelle, de manière moins explicite, "normes et cadences ». Ce sont des vitesses et durées conventionnelles qui sont à l'origine fondées sur des chronométrages, mais elles ont vocation à supplanter ces derniers, car elles doivent permettre de calculer de nouvelles durées sans réaliser de nouveaux chronométrages.

On se propose ici de retracer la genèse de ce dispositif, suivant une démarche historique encore peu développée dans l'étude des outils de gestion (Ghaffari et al., 2013). Ce n'est pas chose facile car, sans même évoquer les dénégations les plus grossières ${ }^{2}$, La Poste refuse la transparence sur les modèles qu'elle utilise. Leur mise en lumière est partie intégrante de la

2. « Nous n'avons pas une organisation mécanique, j'ai entendu des organisations modélisées par des logiciels, non! La Poste, ce n'est pas ça », déclarait la Directrice des ressources humaines de la branche courrier au micro de RTL le 7 octobre 2016. 
bataille qui se joue à leur propos. L'un des nœuds de la controverse actuelle réside dans l'incapacité assumée de La Poste à rendre compte de ses chronométrages initiaux. Cela peut paraître désinvolte pour une aussi grande structure, mais, ainsi qu'on le verra, cela s'explique peut-être par le fait que les temps virtuels utilisés aujourd'hui n'ont pas été établis pour cela. Tôt conçus pour les guichets des bureaux de poste, les «temps standards » ou « temps virtuels » ont été tardivement envisagés pour le courrier. Leur usage a été longtemps repoussé, avant qu'au début des années 2000 un double choc conduise La Poste à un revirement pragmatique, que sa théorie a dû suivre. Mais cette théorie est aujourd'hui de plus en plus vivement contestée par des facteurs et leurs représentants.

\section{Encadré 1}

\section{Méthodologie}

Les interrogations à l'origine de cet article ont pris forme dans des enquêtes de terrain portant sur le travail de facteur dans une douzaine de centres de distribution du courrier de différentes régions de France. Si ces enquêtes informent notamment sur les perceptions et attitudes des facteurs à l'égard des réorganisations et des calculs qui les fondent, le matériau de l'article est essentiellement constitué d'archives, dont la source est systématiquement indiquée en note de bas de page.

Les fonds consultés sont de quatre types : archives nationales (indiquées AN avec la cote du carton); Institut de recherches et prospective postales (Irepp) et Bibliothèque historique des postes et télécommunications (BHPT) ; fonds syndicaux ; avis et analyses de l'Autorité de régulation des communications électroniques et des postes (Arcep), disponibles en ligne sur le site de l'institution.

S'y ajoutent des entretiens avec six responsables nationaux, actuels ou passés, au sein de la branche courrier et des équipes statistiques du groupe La Poste, ainsi qu'un entretien avec un membre de l'Arcep.

\section{De 1946 au début des années 1980 : un outil du guichet que La Poste juge inexploitable pour la distribution du courrier}

\section{I.1. De l'usine au guichet de La Poste, les "temps standards ॥ comme un aiguillon de l'intensité du travail}

À l'usine

Dans sa vaste étude du travail aux usines Peugeot, Hatzfeld (2005) consacre une analyse spécifique à l'effacement progressif du chronométrage des opérations des ouvriers, au profit de l'utilisation de "temps virtuels », précalibrés. Alors que les décennies de l'après-Seconde Guerre mondiale 
avaient vu la généralisation du chronométrage comme moyen d'évaluer et prescrire le travail, le développement de l'informatique dans les années 1980 a permis de déployer la méthode dite des «temps standards », née aux ÉtatsUnis dans les années 1940. Elle consiste à remplacer les chronométrages globalisants d'une opération complexe (réalisés à nouveau dès lors qu'il y a altération ou création d'une opération) par une décomposition en opérations plus simples auxquelles ont déjà été affectées des durées grâce à des chronométrages antérieurs. Il est alors possible d'imputer des durées à des tâches sans les avoir spécifiquement chronométrées. Comme l'écrivent les inventeurs (ou du moins les premiers propagandistes) de la méthode :

«Le processus suivi consiste à identifier entièrement tous les mouvements demandés pour l'exécution de l'opération étudiée, puis à attribuer à chaque mouvement le temps standard prédéterminé qui lui correspond, enfin à faire la somme de ces temps élémentaires pour obtenir le temps standard de l'ensemble » (Maynard et al., 1953:7).

Les chronométrages se sont ainsi raréfiés, devenant davantage un moyen ponctuel de contrôle, voire une revendication des ouvriers et de leurs représentants, contre l'application rigide de durées obtenues par addition de temps prédéterminés. En dehors de l'étude de Hatzfeld, l'ampleur du remplacement des chronométrages par les temps virtuels est peu documentée. Chatzis (1999), après avoir montré que la communauté française des ingénieurs a été tôt convaincue par l'usage des temps standards, suggère que ce dernier a coexisté dans les grandes entreprises avec le recours traditionnel au chronomètre. Il aurait servi plus particulièrement « à calculer le temps pris par des opérations purement manuelles » (Chatzis, 1999:251), avant d'être progressivement rendu obsolète par le développement de l'automation.

\section{Au guichet de La Poste}

Parce que les temps virtuels évoquent, davantage encore que les chronométrages, l'uniformisation des tâches et de leur séquencement, parce qu'ils supposent de mesurer des gestes élémentaires en les isolant des perturbations extérieures, on pourrait penser que les quatre murs d'une usine sont le terrain privilégié de leur déploiement. Comment comprendre alors que La Poste ait décidé de l'appliquer à un métier, celui de facteur, dont l'essentiel de l'activité se déroule en extérieur, dans des environnements variés dont il faut s'accommoder, l'entreprise postale ne pouvant les aménager qu'à la marge ? L'histoire est longue et hésitante.

Les recherches sur le métier de facteur n'ont pas abordé la question des temps standards. Pour beaucoup réalisés à une époque où La Poste avait adopté une politique de recherche volontariste, c'est-à-dire entre les années 1990 et le début des années 2000, ces travaux n’ont pas eu le temps de voir la mise en œuvre de la nouvelle méthode de calibrage des tournées (Gadrey, 2000 ; 
Cartier, 2003), ou bien n'en faisaient que la toile de fond d'un autre sujet (Demazière, Mercier, 2003 ; Demazière, 2005 ; Devetter, Rousseau, 2003). Certains évoquent davantage ce qu'il remplace (Bras, 1995 ; Salaün, 2008) : le chronométrage de chacune des tournées par les «vérificateurs ", les conflits et les soupçons que ce système génère. À l'instar de ce que décrit Hatzfeld pour les chronométreurs de Peugeot, les facteurs contestaient fréquemment les jugements réalisés par les «vérif” " (par exemple sur la définition des bornes d'une séquence de travail à mesurer), tandis que la direction craignait la complaisance de ces derniers, souvent d'anciens facteurs.

Au sein de La Poste, les temps virtuels apparaissent, à l'origine, pour saisir une autre activité que celle des facteurs. En effet, dès 1946, les tâches des guichetiers des bureaux de poste ${ }^{3}$ sont référencées dans ce qui sera appelé jusqu'à la fin du XX $X^{\mathrm{e}}$ siècle la «statistique 539 ». Rebaptisé depuis « référentiel des opérations postales », cet inventaire comprend des centaines d'« opérations » (remettre une lettre recommandée, verser de l'argent sur un livret A, émettre un mandat...) auxquelles sont attribuées des durées, obtenues en additionnant les temps affectés aux « tâches élémentaires » qui composent les « opérations ». La précision des durées va jusqu'au centième de « centiminute », c'est-à-dire 6 millièmes de seconde. Au fil des années, l'inventaire est fréquemment révisé, pour ajouter ou supprimer des « opérations », pour rassembler ou diviser des catégories. Ce qui bouge peu, en revanche, ce sont les quelques centaines de "tâches élémentaires » susceptibles de composer une opération (« accueillir le client », « se faire remettre un document d'identité », « faire signer le formulaire »...), sortes de briques de base qui constituent le matériau uniforme de constructions qui sont quant à elles hétéroclites ${ }^{4}$.

Ce qui change peu, également, c'est l'usage qui est fait de ce référentiel : en multipliant les temps des opérations par le nombre d'opérations recensées pendant une certaine période, on obtient des durées de travail, déclinées par produit, par branche, par bureau de poste, etc. D’un côté, cela informe la comptabilité de gestion de l'entreprise ${ }^{5}$. De l'autre, cela permet de décider d'un niveau d'effectifs, considéré grâce à ce calcul comme nécessaire et suffisant pour écouler la charge de travail qui se présente dans tel ou tel bureau de poste.

Le rôle des temps virtuels dans les bureaux de poste est similaire à celui qu'ils jouent à l'usine : celui d'une norme qui doit aiguiller les efforts des travailleurs, et donc permettre à la direction d'obtenir l'intensité voulue de travail. Lorsque la direction revoit à la baisse les durées attribuées

3. Il est probable que les plateformes de tri (en amont des établissements de facteurs), ou les ateliers de production des services financiers (saisie des chèques par exemple) aient fait l'objet de rationalisations analogues, mais je n'ai pas mené l'enquête sur ce point. Dans les archives consultées, le cas du guichet était présenté comme une référence pour la branche courrier.

4. Source : AN 20050328/5, 20000508/28 et $19980486 / 5$, et sources syndicales.

5. Cela permet aujourd'hui la facturation interne de ses prestations par la branche « Réseau » (les bureaux de poste) aux autres branches du groupe La Poste, notamment le courrier et la banque. 
aux mêmes opérations, elle cherche à intensifier le travail, c'est-à-dire à accroître l'usage qu'elle peut faire des travailleurs pendant le temps où ils lui sont subordonnés. Mais la quantification préalable du travail est enserrée dans un cadre horaire fixe - le bureau de poste a des horaires déterminés d'ouverture et de fermeture -, si bien que l'écart éventuel entre la quantité produite et la norme fixée reste à la charge de l'employeur ${ }^{6}$.

Il en va autrement des temps virtuels auxquels sont aujourd'hui soumis les facteurs, puisqu'ils ne contribuent pas seulement à définir un niveau d'effectifs à l'échelle d'une unité de production, mais aussi à assigner à chaque travailleur une charge de travail qu'il devra écouler intégralement. C'est alors le temps de travail du facteur qui devient la variable d'ajustement, et non la production, qui demeure intouchable - il faut chaque jour distribuer tout le courrier du jour (voir infra).

\section{I.2. Une invention dont doutent ses inventeurs}

\section{« L'application de normes trop précises aboutirait rapidement à une rigidité paralysante »}

Du côté de la distribution du courrier, les temps standards sont plus jeunes que ceux des guichets. Non que l'idée ait manqué : les dirigeants et ingénieurs de la branche courrier sont au courant de ce que font ceux du « réseau » des bureaux de poste, d'autant que ce sont parfois les mêmes. Mais, jusqu'aux années 1980, les archives disponibles laissent percevoir un débat interne sur le bien-fondé d'une telle méthode appliquée à la distribution du courrier. Sinon, pourquoi la Direction générale des Postes ressentirait-elle le besoin d'affirmer, en 1972, que, « dans un domaine aussi mouvant que celui de la distribution, fortement influencé par la topographie des lieux, par la structure de l'habitat, par le milieu social ou économique, l'application de normes trop précises aboutirait rapidement à une rigidité paralysante ${ }^{7}$ » ? À qui croit-elle utile de rappeler ce constat lorsque, 15 ans plus tard, elle écrit qu'« en raison des nombreuses et inévitables disparités existant sur le terrain, dues à des comportements humains différents ou résultant de particularités géographiques, chaque situation ne peut valablement être appréhendée qu'en prenant en compte des spécificités locales, rendant par-là quasiment inopérante une éventuelle méthode universelle applicable dans tous les cas de figure ${ }^{8}$ »? Les sources étant peu bavardes, il est difficile de savoir quelles ardeurs ces affirmations cherchent à tempérer, ou quelles peurs elles entendent apaiser.

6. Deux précisions : 1) Cela ne veut pas dire qu'il n'y ait pas de sanction pour les travailleurs qui ne respecteraient pas le quota. Mais, sur le moment, sauf cas (devenu) exceptionnel de rémunération au rendement, le coût de l'écart entre la norme fixée et la quantité produite est assumé par l'entreprise. 2) L'entreprise peut avoir une stratégie délibérée de réduction de la production, de telle sorte que cet écart à la norme ne lui pose pas problème. On peut penser que c'est le cas pour une partie des bureaux de poste, où la direction souhaite réduire les contacts avec la clientèle, notamment la plus défavorisée.

7. Direction générale des postes, La Poste face à la mécanisation du tri, 1972, p. 31. AN 19930274/6.

8. La Poste, Études d'organisation de la distribution postale, 1987, p. 6. BHPT. 
Comme l'indique La Poste dans ces années-là, les conditions d'exercice du métier de facteur déjouent les tentatives de modélisation parce que leur variabilité est forte d'un lieu ou d'une période à l'autre. Elle tient au relief, à la météo, à la densité et au type de la population, des habitations et des circulations, à la qualité de la nomination des voies et de la numérotation des logements, aux migrations pendulaires (déterminant la disponibilité des usagers lors de la distribution) et touristiques (impliquant des variations saisonnières plus ou moins fortes), etc. Elle se prête difficilement à une rationalisation uniforme telle qu'elle a pu être conçue entre les murs des usines (et déjà contestée dans ce cadre, comme le montre Hatzfeld).

Il semble donc que les facteurs sont voués à avoir un chronométreur derrière eux, nommé à La Poste "vérificateur ». C'est coûteux car, à la différence d'une usine où les postes de travail se ressemblent, chaque facteur, premier arpenteur du territoire, épouse sur «sa » tournée les spécificités de la zone à laquelle il est dédié. Il faut donc que le vérificateur, au cours de ce qu'on appelle à La Poste des " accompagnements », chronomètre chaque facteur, ou plutôt chaque tournée (parfois plusieurs fois, avec différents facteurs). Les accompagnements conduisent à déterminer la durée des différentes portions de la tournée (qui, s'additionnant, établissent la durée de la tournée). Puis, à la faveur d'une "réorganisation ", le vérificateur raccourcit ou au contraire rallonge le parcours afin de faire correspondre la durée de la tournée et la durée réglementaire du travail.

\section{Vérifier le travail des « vérificateurs »}

Pourtant, entre 1972 et 1987, c'est-à-dire entre les deux citations convergentes de la direction de La Poste, cette même direction abrite (et probablement suscite) des études qui cherchent à fournir aux durées chronométrées l'escorte nouvelle de durées que La Poste qualifie alors de «théoriques ».

En 1983, la Direction des services postaux édite une Évaluation de l'organisation extérieure de la distribution ${ }^{9}$ qui construit, à partir d'un échantillon de 1620 tournées, une analyse factorielle de correspondances. Au-delà de l'effet de mode dont jouit une telle technique à la fin des années 1970 (Bonnet, 2015), il s'agit de repérer quels sont les principaux déterminants de la durée d'une tournée ${ }^{10}$, afin de construire ensuite une typologie de tournées à partir desquelles seraient construites des durées théoriques. Le document

9. Source : AN 19930274/6.

10. L'étude aurait « mis en évidence le rôle prépondérant de : la longueur du parcours actif [c'est-àdire de la distance pendant laquelle le facteur doit distribuer du courrier] ; le nombre de points de distribution [ce qui correspond à une maison, un immeuble, une adresse]; le nombre de points de remise [les boîtes aux lettres], à l'exclusion des données de trafic en objets ordinaires » (p. 15). Une telle conclusion est aujourd'hui subversive : alors que la direction de La Poste justifie la suppression massive et continue de tournées par la baisse du courrier, cette étude affirmait donc que le lien entre volume de trafic et temps nécessaire pour l'écouler est faible ou inexistant. Mais à l'époque de l'étude, alors que le volume de courrier ne cessait d'augmenter, la conclusion correspondait davantage aux intérêts de la direction, en minorant les besoins en effectifs découlant de cette augmentation. 
ne prétend pas substituer ses raisonnements aux chronométrages effectués sur le terrain par les " vérificateurs », mais fournir un repère supplémentaire (une " durée moyenne théorique ») pour évaluer la qualité des projections établies par chronométrage. Tant sur le plan de la technique de modélisation que de l'usage préconisé pour cette technique, cette tentative se distingue des solutions qui seront adoptées 20 ans plus tard, et parfois les critique déjà.

Sur le plan technique, l'étude critique le recours aux temps standards appliqués à des tâches artificiellement séparées par une parcellisation purement intellectuelle, recours qui semble avoir déjà été tenté : «On avait essayé de fabriquer une statistique 539 "distribution" [référence aux temps standards du guichet, voir supra] en utilisant des concepts qui, ayant fait leurs preuves au niveau de la 539 pour le service général, étaient jugés capables de rendre les mêmes services pour résoudre les problèmes du "vide statistique" de la Distribution. En fait, cette tentative n'a pu aboutir parce qu'elle (...) procédait d'une décomposition des activités des préposés [facteurs] en tâches élémentaires qui laissaient de côté les éléments non paramétrables tels que l'activité de contact avec la clientèle et dénaturaient ainsi l'analyse du problème ${ }^{11}$. »

À l'encontre d'une telle démarche, l'étude entend obtenir la "durée moyenne théorique » d'une tournée non plus par l'addition de tâches et de durées élémentaires, de morceaux de tournée, de gestes qui s'enchaînent, répertoriés dans des tableaux avec des seuils séparant des valeurs constantes et étanches, mais globalement au moyen de fonctions mathématiques impliquant des relations continues entre variables (durée, longueur du parcours, densité des habitations...) adaptées par type de tournées ${ }^{12}$. L'étude établit que la durée de distribution est une fonction non linéaire de la longueur du parcours. Dans cette perspective, il n'est alors pas possible d'assigner des durées à des petites portions de parcours et ensuite d'agencer ces dernières de manière à construire des tournées, puisque l'addition des durées des portions de parcours ne serait pas équivalente à la durée calculée pour l'ensemble du parcours constitué par ces différentes portions. Contrairement aux constructions ultérieures, l'hypothèse que retient ici La Poste est que le tout n'est pas égal à la somme des parties.

Le choix technique interdit ainsi toute construction des tournées, toute définition des effectifs nécessaires qui partirait de rien. Il permet d'évaluer la durée théorique d'une tournée seulement une fois que celle-ci est dessinée, et il s'agit alors de mesurer l'écart avec la durée retenue par le « vérificateur ».

Sur le plan des usages, quoique la méthode le permette (et même le requière comme une étape intermédiaire), il ne s'agit pas d'évaluer la justesse

11. Ibid.

12. « II apparaît donc utile, et à coup sûr plus proche de la réalité, de prendre en compte la totalité de la tournée pour effectuer le calcul de la DMT [durée moyenne théorique] et d'embrasser ainsi tous les aspects du métier de distributeur. L'évaluation de la DMT est faite sur l'ensemble de la tournée et de manière spécifique pour chaque famille typologique » (ibid., p. 13-15). 
d'une tournée prise isolément. C'est un repère, un moyen de contrôle à un niveau agrégé, celui d'un établissement, c'est-à-dire de plusieurs dizaines de tournées, pour voir si, globalement, il n'y aurait pas un écart préoccupant entre les durées retenues par les « vérificateurs » (dont sont déduits le nombre total de tournées et les besoins en effectifs) et celles indiquées par le «modèle statistique explicatif » construit par l'étude. Le modèle est en effet à destination " des chefs de service départementaux et régionaux ${ }^{13}$ ", afin qu'ils évaluent le travail de leurs subordonnés, c'est-à-dire l'ensemble de l'organisation conçue par un «vérificateur » et validée par son directeur d'établissement.

En dépit des divergences techniques avec les solutions ultérieures, cette étude traduit la volonté d'amorcer un nouveau mode de contrôle des encadrants de premier niveau, surveillés par un encadrement de niveau supérieur lui-même tenu par des formules conçues par une Direction générale qui tente ainsi d'accroître sa maîtrise des opérations. Cependant, l'étude de 1983 ne semble pas avoir émergé des limbes de la littérature grise et atteint le stade de l'application opérationnelle. Elle a été rapidement supplantée par d'autres logiques de rationalisation - celles-là mêmes qu'elle critiquait.

\section{De la fin des années 1980 au début des années 2000 : des objectifs ambigus pour la fabrication de temps standards}

Dans les années 1990, une série d'initiatives accélère la modélisation des tournées des facteurs. Il n'est pas facile de déterminer comment s'articulent ces initiatives, car les documents qui en rendent compte ne se réfèrent pas toujours les uns aux autres. Des motifs à la fois opérationnels (organiser le travail) et comptables (calculer le coût de l'acheminement des différents produits) contribuent à l'élaboration des temps standards, sans que la nature de leurs relations puisse être entièrement explicitée.

\section{II.1. "Exploiter les données théoriques et celles recueillies sur le terrain »}

Sur le plan opérationnel, La Poste resserre son emprise sur les « vérificateurs ", par la diffusion de nouvelles consignes et par le contrôle des produits de leur activité, c'est-à-dire les parcours et la durée des tournées. Elle le fait selon un mode qui contredit les réflexions et préconisations de l'étude de 1983 examinée ci-dessus.

Tout d'abord, en 1987, La Poste édite une «méthodologie générale de construction de tournées et des organisations du service de la distribution » à destination des "vérificateurs », " un guide pour faciliter la tâche des agents chargés $(. .$.$) de procéder au découpage des tournées { }^{14}$ ». Pour 
la première fois, des cadences et des temps standards sont indiqués. Le document ne précise pas les circonstances et modalités de leur conception. Cependant, d'une part, ces cadences ne concernent qu'une partie du travail du facteur : les « travaux intérieurs » (le tri des plis au sein de l'établissement avant le départ en tournée ${ }^{15}$ ), les «temps forfaitaires » (pause, vérification de sécurité sur le véhicule, reddition des comptes ${ }^{16} \ldots$...) et la remise des " objets spéciaux » (lettre recommandée, mandat...). Les temps de parcours, d'arrêt, et la remise des " objets ordinaires » (c'est-à-dire le toutvenant des lettres), qui constituent le plus gros de l'activité, ne font l'objet d'aucun calibrage. D'autre part, ces cadences et durées ne constituent pas une prescription. Ce sont des « correctifs », qui permettent de jauger mais pas de remplacer les durées relevées sur le terrain. Ces temps standards servent au vérificateur de « point de comparaison » et à ses responsables hiérarchiques de moyen de contrôle de son travail ${ }^{17}$.

Ensuite, un projet vise à « mettre au point un indicateur permettant d'apprécier les performances » des différentes organisations de la distribution. Le document qui en fait état ${ }^{18}$ semble dater de 1993, mais il ne mentionne ni la date ni le service qui en est l'auteur. Comme dans l'étude de 1983, il s'agit de forger un indicateur permettant de mesurer un écart entre le dimensionnement actuel et le dimensionnement théorique des tournées (et donc des effectifs). En revanche, sur le plan technique, le document annonce la généralisation de la méthode du guide de 1987, que l'étude de 1983 remettait en cause : la " décomposition des activités des préposés en tâches élémentaires ». Au lieu de calculer une durée globale pour la tournée, elle la diviserait en « unités d'œuvre », appelées ici « unités de charge distribution » (ou « UCD », l'acronyme désignant en même temps le logiciel qui en manipulerait les informations). Pour les travaux intérieurs, ce sont les cadences de tri qui sont utilisées ; pour les « travaux extérieurs » (la distribution), il s'agirait d'une combinaison de « vitesses moyennes de déplacement selon le moyen de locomotion » et de «temps de remise » (qui font l'objet de «standards différents selon le type d'objet distribué »). Le document indique que cette «modélisation des travaux intérieurs et extérieurs » serait réalisée en 1987, mais les standards utilisés n’y sont pas reproduits.

15. À la différence des autres cadences, celles concernant le tri sont comprises dans des fourchettes, conférant une marge de manœuvre aux directions locales pour déterminer le niveau qui leur semble adéquat au sein de ces fourchettes.

16. À son retour de tournée, le facteur rend ses comptes, c'est-à-dire éventuellement l'argent récolté par ses ventes de timbres, mais surtout il restitue les recommandés non distribués qui seront alors mis en instance.

17. Ibid., p. 13. À noter que le guide fait une allusion fugace à l'étude de 1983 et son " modèle statistique explicatif », en suggérant timidement que l'on peut comparer les durées établies pour les tournées avec les durées théoriques données par le modèle : "Par ailleurs, il peut être intéressant afin de compléter le dossier, d'utiliser le modèle statistique explicatif pour estimer la validité globale de l'organisation envisagée. En fonction du diagnostic établi, il sera alors encore temps de procéder à de dernières rectifications » (p. 22). On a connu - et on connaîtra formulation plus prescriptive.

18. L'unité de charge distribution, AN 20060660/14. 
À ce stade, il ne s'agit toujours que d'un moyen de contrôle de second degré, et non d'un outil employé directement au découpage des tournées. «L'approche n'est pas concurrente des calculs d'organisation. Elle ne prétend pas être un outil d'organisation mais bien une aide à la gestion. (...) L'UCD ne doit pas être un jugement dans l'absolu mais corroborer d'autres approches ${ }^{19}$.» Cela explique que le compte-rendu d'une réunion de $1994^{20}$ ayant pour objet de «fixer le contenu de la formation à l'UCD » indique que les cibles de la formation sont le "personnel impliqué dans l'arbitrage d'un contrat de gestion », c'est-à-dire « en priorité » les « responsables départementaux de la distribution », et dans un second temps les « inspecteurs de l'organisation ", qui sont en quelque sorte les vérificateurs des vérificateurs. À l'instar du «modèle statistique explicatif » de 1983, l'UCD n'est encore considérée que comme un moyen d'éprouver les découpages opérés par les vérificateurs (qui demeurent fondés sur des chronométrages) et les besoins en force de travail qui en découlent.

Pourtant, rapidement, tant le statut des «vérificateurs » que le contenu de leur activité sont repensés. Un nouveau métier, celui d'« organisateur », est créé et supplante progressivement les "vérif" ». Alors que l'UCD était conçue, en 1994, comme un moyen de contrôler le travail du vérificateur, elle apparaît dès 1995 comme un outil de l'« organisateur » lui-même : «L'organisateur doit être capable d'analyser les résultats de l'UCD ${ }^{21}$.» S’il faut qu'il en soit capable, c'est parce qu'il doit désormais « effectuer les calculs du temps nécessaire aux travaux intérieurs et extérieurs des facteurs, en exploitant les données théoriques et celles recueillies sur le terrain ${ }^{22} \%$. Nouveau glissement : les «données théoriques » ont quitté le statut de « correctifs », de « pondérations » qu'elles avaient en 1987, et sont désormais à égalité avec les chronométrages.

\section{II.2. Un sous-produit de la comptabilité analytique}

Sur le plan comptable, c'est également l'heure des grandes manœuvres. La réforme des PTT de 1990 prépare l'ouverture à la concurrence en contraignant La Poste à établir et communiquer une « comptabilité analytique » (ce que l'on nomme également, aujourd'hui, comptabilité de gestion). Une comptabilité analytique consiste à établir les coûts de revient des différents produits. Dans des processus souvent continus et mixtes - le facteur distribue dans un même parcours le pli ordinaire et le recommandé, la lettre simple et le colis, le pli urgent et l'économique -, la comptabilité analytique cherche, par un système de définitions, de conventions

20. UCD, Compte-rendu de la réunion du 17 février 1994. AN 20060660/14.

21. Organisateurs distribution, Compte-rendu de la réunion du 22 novembre 1995. AN 20060660/14 22. Ibid. 
et d'équivalences, à découper et valoriser des coûts qui reviendraient en propre à chaque objet.

Dans les entreprises classiques, toutes les mesures qui vont au-delà des quelques exigences réglementaires du plan comptable général constituent un outil conçu par et pour les directions, afin d'informer leur réflexion stratégique. Dans le cas de La Poste, l'usage n'est pas qu'interne : c'est un tableau qu'elle est obligée de produire, selon des règles dont elle ne peut décider seule, afin que les autorités de régulation du secteur postal puissent à l'avenir garantir l'aspect concurrentiel de ce nouveau marché. L'ouverture à la concurrence étant progressive, il ne faudrait pas, dans l'esprit de la réforme, que La Poste profite du monopole qu'elle conserve sur certaines activités pour pousser son avantage sur celles qui sont désormais disputées. Prenons l'exemple du produit le plus tardivement livré à la concurrence, la lettre de moins de 50 grammes : le facteur la distribue en même temps qu'il distribue des colis, dont le marché est ouvert à la concurrence ; il convient alors de distinguer ce qui, dans son parcours et ce qu'il coûte, est imputable à la lettre et ce qui l'est au colis. Cela doit permettre d'ajuster la tarification des différentes correspondances et d'éviter toute "subvention croisée ", selon l'expression consacrée. L'opération est affaire de conventions, ésotériques pour le facteur qui distribue tout d'un même mouvement, mais décisives pour la régulation du secteur postal jusqu'à aujourd'hui.

En vertu de cette obligation, La Poste dessine en 1992 un "schéma directeur de la comptabilité analytique ». D'après ce document, jusque-là l'information comptable remontait peu des établissements locaux. L'estimation des coûts de revient se faisait au moyen de gros chiffres, par agrégations massives de quelques données (effectifs, trafic...) et applications de moyennes nationales. Les chronométrages locaux des vérificateurs servaient à organiser la production mais pas à informer la comptabilité. L'objectif, désormais, est « de passer de coûts de revient nationaux établis à partir de moyennes et de statistiques nationales à des coûts de revient nationaux établis par combinaison de coûts de revient locaux, eux-mêmes établis sur la base de coûts de production liés à la structure de la production et aux mesures locales de l'activité ${ }^{23} »$.

Il ne faut pas se tromper : cette attention au local implique que le national impose son étalon de mesure. Pour que l'information locale remonte au national, il faut que le national étende son emprise, par la diffusion de catégories de saisie. Il faut que « quels que soient les moyens de production spécifiques aux unités de production, les opérations élémentaires nécessaires au calcul du coût du produit soient uniformisées ${ }^{24} »$. Lorsque le national parle de «mesures locales de l'activité », il n'attend pas que les

23. Présentation du schéma directeur de la comptabilité analytique de La Poste. AN 19980486/5 24. Schéma directeur de la comptabilité analytique. Projet, p. 27 . AN 19980486/5. 
facteurs, ou même leurs chefs d'équipe, ou même leurs « vérificateurs » qui sont en train de devenir des « organisateurs », rendent compte de l'activité dans leurs propres termes ; il entend que cette activité soit représentée et valorisée par la combinaison réglementée de variables (volume et types de courrier, longueur du parcours, densité des habitations) et de normes standardisées de vitesse.

\section{II.3. 1994 : les cadences d'aujourd'hui créées pour les besoins de la comptabilité}

Cette même année 1992, le directeur du courrier commande à la Direction de l'informatique du courrier une « étude déterminant les temps de remise par catégorie d'objet et par type de tournée ainsi que les temps de parcours », " pour me permettre de mettre à jour les éléments mis en œuvre par la Direction des affaires comptables, juridiques et fiscales ${ }^{25} »$ - cellelà même qui conçoit le "schéma directeur de la comptabilité analytique ». Le cahier des charges établi un an plus tard assigne à l'étude cet objet : «Actualiser les temps de parcours et de remise des travaux extérieurs, ceux utilisés actuellement datent de près de vingt ans ${ }^{26}$. »

L'étude est menée en quelques semaines, pendant l’hiver 1993-1994. Elle consiste dans le chronométrage (selon des modalités non explicitées) d'un échantillon de tournées (dont le nombre n'est pas précisé) au sein de 20 bureaux de France métropolitaine. Dans une première étape, l'étude établit des vitesses et des temps standards de distribution. Par exemple, il est dit qu'un facteur cycliste roule à $6,19 \mathrm{~km} / \mathrm{h}$ lorsqu'il y a entre 4,6 et 6 points de distribution sur une distance de 100 mètres, pendant qu'une voiture avance à $7,32 \mathrm{~km} / \mathrm{h}$; ou qu'un automobiliste « séjourne » 52,37 « centiminutes » (hors temps de remise des lettres) à une adresse qui compte entre 3 et 9 boîtes aux lettres ; ou encore, qu'il faut 3,78 " centiminutes » pour remettre une lettre dans une boîte aux lettres insérée dans une batterie de 9 à 12 boîtes.

Que sont supposées représenter ces valeurs ? Pour la vitesse, il est dit qu'il s'agit de la « vitesse moyenne », mais est-ce à dire que le chiffre retenu est la moyenne de tous les chronométrages, sans pondération ${ }^{27}$ ? C'est

25. Courrier du 6 juillet 1992, annexé à l'Étude sur les travaux extérieurs de la distribution des objets ordinaires, La Poste, Direction du courrier, septembre 1994. Source syndicale.

26. Cahier des charges : durées des travaux extérieurs à la distribution, annexé à l'Étude..., op. cit. La datation est curieuse. Elle fait écho peut-être aux tentatives que critiquait l'étude de 1983 , mais semble oublier la " modélisation des travaux intérieurs et extérieurs " de 1987 sur lesquelles l'UCD était supposée se reposer (à moins que cette modélisation n'ait été qu'un projet que la présente étude viendrait justement actualiser).

27. La question du choix de la vitesse de référence parmi des relevés disparates a toujours donné lieu à débat parmi les ingénieurs de la décomposition des temps et des mouvements, débouchant sur des solutions différentes. En France dans les années 1950, le « Bureau des temps élémentaires » recommandait de « prendre pour vitesse de référence la vitesse ayant la plus grande fréquence (ou mode) au sein de la distribution des vitesses observées dans une population de travailleurs » (Chatzis, 1999:242). 
peu probable. Quant au «temps de séjour au point de distribution » et au « temps de remise » des plis, il n'est même pas précisé s'il s'agit de temps moyens. Le document n'explicitant pas sa méthode et ne présentant pas de relevé des observations effectuées (La Poste affirmant n'en avoir conservé aucune trace, comme on le verra), il est impossible de déterminer comment des résultats nécessairement hétérogènes ont été convertis en des vitesses ou durées uniques.

Sans que le lecteur puisse savoir ce qui fonde la précision des résultats, certains d'entre eux sont présentés comme approximatifs : «En raison de difficultés techniques, les tournées "cyclomoteur" ont été suivies en voiture et les prises de données n'ont pu être menées de façon aussi précise. En conséquence, les valeurs calculées sont-elles données à titre indicatif ${ }^{28}$. Peut-être cette approximation est-elle tolérée dans la mesure où l'étude a essentiellement pour objet d'améliorer l'information comptable. Il peut être admis qu'une telle information est, nécessairement, une représentation simplifiée et imprécise de processus de production complexes.

Les vitesses et les durées auxquelles les facteurs sont supposés travailler sont enregistrées dans une compilation de quelques tableaux. Ils déclinent la vitesse de déplacement, le «temps de séjour au PDI » (la durée passée à une même adresse), et le "temps de remise d'un objet » en fonction de la densité des habitations, du nombre de boîtes aux lettres à une même adresse et du mode de locomotion. Dans chaque case, un seul chiffre : pas d'indicateur de dispersion, pas d'intervalle de confiance, mais une cadence unique. Cette compilation, insérée au milieu du rapport, est décisive : quoiqu'ils datent de 1994, ce sont les mêmes chiffres qui, aujourd'hui, gouvernent l'activité des facteurs.

Pourtant, ces résultats ne sont alors qu'un sous-produit de l'étude, une étape intermédiaire pour atteindre son objectif : déterminer le coût de revient de chaque lettre distribuée. Les tableaux finaux de l'étude indiquent ainsi le temps de travail nécessaire à la distribution d'un objet ordinaire selon le type de tournée et de moyen de locomotion. Par exemple, lorsqu'on additionne les temps de déplacement, de séjour et de remise, il faudrait à une tournée rurale réalisée en voiture 37,45 centiminutes pour remettre une lettre, tandis qu'il en faudrait 8,93 à une tournée urbaine réalisée à vélo dans une zone de petits bâtiments ${ }^{29}$. Si les cadences attribuées au travail des facteurs sont théoriques, ces durées de distribution par objet sont carrément fictives, ou du moins conventionnelles, dans le sens où elles ne prétendent pas correspondre à une opération concrète : on ne distribue jamais un seul objet, et si on le faisait cela prendrait bien plus de temps.

29. Tous ces chiffres sont issus de l'Étude..., op. cit. 


\section{II.4. Une boîte à outils désormais complète}

Cinq ans plus tard, en 1999, La Poste réédite le Guide méthodologique de l'organisation de la distribution qu'elle destine aux «vérificateurs/organisateurs » et à leurs encadrants. Cette fois, le document propose des durées théoriques pour tous les compartiments de l'activité, travaux intérieurs et extérieurs. Pour ce faire, il a intégré les cadences définies par l'étude de 1994 (ou, plus précisément, son sous-produit, qui était nécessaire à l'établissement de durées de travail par objet distribué).

Alors que l'étude source de 1994 est à peu près muette sur cette question, le guide de 1999 présente l'ensemble des valeurs comme des « moyennes", sans préciser de quelles observations ont été déduites de telles moyennes. Au fil du texte, la notion de "moyenne » se révèle être une définition unilatérale et autoréférentielle de la cadence attendue, suivant en cela la tradition des inventeurs étatsuniens des temps standards. En effet, ces derniers écrivaient : «Dans l'établissement des définitions, une attention particulière fut donnée à l'activité "moyenne". Il fut décidé qu'elle serait l'équivalent du fameux "travail loyal d'une journée". Cela devait représenter un niveau d'effort pouvant être maintenu aisément bon an, mal an, par l'opérateur physiquement normal, sans nécessiter en aucune façon l'utilisation de réserves d'énergie. Ce devait être l'effort donné par le travailleur consciencieux quand il travaille » (Maynard et al., 1953:7). Tandis que, dans son guide de 1999, La Poste avance, d'une manière qui cache encore plus mal le fait que la norme correspond à l'exigence patronale : «Les cadences proposées représentent ce que l'on est en droit d'attendre comme productivité de la part d'agents titulaires d'une position de travail ${ }^{30}$. »

Avec cette réédition du guide, toutes les cases du tableau sont désormais remplies : il n'y a plus aucune activité du facteur qui ne soit saisie par des temps standards, et donc justiciable d'un calcul de "durées théoriques » permettant de se passer des chronométrages. Le choix de décomposer les tâches du facteur et les séquences de la tournée, que l'auteur de l'étude de 1983 jugeait ruineux, permet théoriquement de construire des tournées de toutes pièces, sans chronométrage, par l'addition de "portions de voie» auxquelles sont affectées des durées calculées par simple combinaison des cadences, des éléments géographiques référencés et du trafic estimé.

Toutefois, le guide de 1999 reste sur la même ligne que ses prédécesseurs : les temps standards ne sont encore présentés que comme un «calcul théorique » dont l'usage doit se limiter à celui d'un point de comparaison : «Les vitesses et normes (...) n'ont en aucun cas valeur de normes officielles, mais seulement une valeur indicative ${ }^{31}$. " Ainsi, à la fin des

30. Ibid., p. 52.

31. Direction courrier, Guide méthodologique de l'organisation de la distribution, 1999, p. 62. Source syndicale. 
années 1990, l'outil des temps standards existe, après des décennies de débats, des tâtonnements, et l'accélération finale commandée notamment par des exigences comptables. Mais leur usage direct, comme moyen de conception des tournées (et non plus seulement de vérification ex post) demeure, sinon proscrit, du moins repoussé. La Poste proteste encore que ces cadences ne sont pas ce qu'elles semblent être, c'est-à-dire le moyen de prescrire le travail des facteurs sans observer leur activité. Elle y croit peutêtre ; mais elle aura bientôt fini d'y croire.

\section{Des années 2000 à aujourd'hui : l'instrument de restructurations accélérées et l'objet de contestations croissantes}

\section{III.1. 2005 : la " modélisation » remplace le chronométrage}

Le nouveau siècle fait disparaitre la prudence, sinon la gêne, que La Poste exprimait à l'égard des temps standards. Désormais, il n'est plus question de dire qu'ils sont juste un moyen d'évaluer des coûts de revient ou d'accompagner l'analyse de l'organisation. Il n'est même plus question de traiter à égalité chronométrages et temps standards. Ces derniers étaient indicatifs, ils deviennent impératifs. Les durées théoriques remplacent les temps chronométrés en devenant le moyen exclusif d'évaluer la charge de travail et de la répartir. La durée des portions des tournées est désormais uniquement calculée, modélisée, en combinant le trafic estimé, les caractéristiques recensées de la tournée, et les «normes et cadences » standards issues de l'étude de 1994. La nouvelle édition du guide méthodologique des organisateurs, datée de 2005, traduit ce changement de cap : « La valorisation des travaux extérieurs se fait par modélisation (...). Elle permet $(. .$. de réorganiser les centres courrier sans accompagnement et sans relevé géographique systématique. La réactivité des organisateurs devient dès lors plus importante ${ }^{32}$. » La bascule est consolidée par l'achèvement du remplacement des « vérificateurs » par les « organisateurs ». Cela n’implique pas seulement un changement de nom et de fonction, mais aussi de profil de recrutement, comme l'explique un responsable national de l'époque : «Les vérificateurs ne sont pas passés spontanément... Il y en a qui n'ont pas réussi à basculer. Généralement, c'étaient des facteurs à l'origine, qui sont montés, donc ils étaient proches de la retraite. Donc l'évolution s'est faite par une rotation naturelle des effectifs, et à la place il y a eu l'embauche d'ingénieurs de logistique - ce sont les meilleurs profils, ils ne sont pas tous

32. Direction courrier, Guide de références de l'organisation de la distribution, 2005, p. 35. Source syndicale. 
comme ça, mais ce sont les meilleurs profils qu'on a recrutés pour cette fonction. »

$\mathrm{Au}$ regard des tergiversations antérieures, la rupture paraît soudaine. Comment l'expliquer ? La traditionnelle méfiance de la direction à l'égard des "vérif'» ne suffit pas, puisque, justement, elle est ancienne et ne rend pas compte du moment choisi pour s'en passer définitivement. La perspective de réaliser des économies a pu jouer, dans un contexte où La Poste se dirigeait vers le statut de société anonyme et peut-être, si l'on se fonde sur le destin de sa jumelle France Télécom, une ouverture de son capital (Adam, 2010) - pour l'instant repoussée. Dans ce contexte, la direction de La Poste a pu envisager que les temps standards soient susceptibles de générer des gains de productivité et une réduction des effectifs de facteurs plus importants que ceux permis par les chronométrages (ou en tout cas une meilleure anticipation et une meilleure maitrise de ces gains par la Direction générale puisque découlant de calculs automatisés). A minima, les temps standards permettent une augmentation de la productivité sur l'activité même de découpage des tournées.

Mais surtout, deux chocs externes ont forcé le basculement. Le premier, c'est la réduction de la durée légale du travail, qui secoue l'ensemble des organisations du courrier - plus que dans d'autres secteurs, puisque le périmètre de chaque tournée est défini par équivalence avec une certaine durée, elle-même calibrée en référence à la durée légale du travail. La direction de La Poste souhaite compenser intégralement le passage aux 35 heures par une augmentation de la productivité de 10\% (Samzun, 2007:355-356).

Le second choc, c'est la rupture de la tendance à la hausse du volume de courriers, tendance qui était continue depuis la fin de la Seconde Guerre mondiale. Il ne se passe qu'une poignée d'années entre les signes avantcoureurs - le volume de plis cesse de croître au même rythme que le PIB à la fin des années 1990 -, la stagnation, et finalement la décrue incessante depuis la fin des années 2000. Alors que La Poste distribuait 18 milliards d'objets en 2000, elle en distribue un tiers de moins 15 ans plus tard (Cour des comptes, 2016b:86).

Ces deux chocs, l'un ponctuel, l'autre structurel, conduisent La Poste à revoir simultanément nombre d'organisations existantes. Auparavant, « faire de la productivité », selon l'expression indigène, consistait à produire plus avec autant de moyens, ou le moins de moyens supplémentaires possible. Le volume de courrier augmentant constamment, la sacoche du facteur s'alourdissait mécaniquement (Gadrey, 2000). Quand il y avait une réorganisation, c'était plutôt à la demande des agents, pour alléger leurs tournées. À partir du moment où le volume de courrier fléchit, « faire de la productivité » consiste à produire moins avec encore moins de moyens. Les réorganisations sont alors à l'initiative de la direction et de plus en plus 
fréquentes, afin de supprimer des tournées et " recharger » les tournées restantes. Un directeur de La Poste, alors en charge des questions de modélisation au siège, résume ainsi le raisonnement adopté face aux deux chocs : « Il y a eu le choc des 35 heures. Il a fallu redesigner toutes les tournées de France d'un seul coup. Tout d'un coup on avait ce mur à franchir. Côté managérial, on commençait par croire que le courrier allait baisser. Quand le trafic croît et que vous ne réorganisez pas très vite, il faut être honnête, la productivité se fait toute seule. De facto on n'était pas sous le stress de réorganiser fréquemment. Comme on a cru que la courbe de trafic allait s'inverser, il fallait aller chercher la productivité. Il y avait donc deux phénomènes, ce choc des 35 heures, ils le voulaient tous tout de suite; et la baisse du trafic à moyen terme. Du coup on a trouvé que cette idée n'était pas totalement stupide, d'éviter le parcours physique de l'organisateur. »

Il faut donc « aller chercher la productivité », mais au moment où celleci se dérobe. La révision des tournées et l'accroissement de leur périmètre ne peuvent pas compenser entièrement la baisse du trafic puisque, même si les usagers reçoivent moins de courrier, il faut toujours se rendre chez eux pour le leur remettre. L'étude de 1983 n'avait-elle pas exclu le volume de courrier comme variable explicative de la durée d'une tournée, estimant que la longueur du parcours et la densité des habitations étaient des facteurs prépondérants ? Plus récemment, les études d'économie postale s'accordent sur l'idée que la quantité de travail requise pour distribuer le courrier varie moins fortement que le trafic (Cazals et al., 1997, 2005 ; Roy, 1999). La diminution des volumes de courrier devait se traduire, en toute logique, par une baisse de la productivité. On mesure alors l'intensité des restructurations qui ont permis que La Poste soit saluée par la Cour des comptes (2016b) - qui en demande toutefois encore plus - pour les gains de productivité et la réduction d'effectifs réalisés dans la branche courrier.

\section{III.2. Les effets sur l'activité des facteurs : allongement de la durée du travail ou intensification}

Dans les bureaux, les calculs permis par les temps standards parviennent aux facteurs sous l'aspect de « diagnostics » de leurs tournées. Ces diagnostics prennent la forme d'un tableau, qui reprend les grandes catégories de travaux d'une tournée (en distinguant principalement le tri et la distribution), restitue le temps qui est attribué à chacune au sein d'une journée supposée moyenne, et en fait la somme. Dans le langage utilisé dans les centres courrier, si cette addition est inférieure à la durée réglementaire de la journée de travail, alors le facteur « doit » du temps à La Poste, ce qui justifie un allongement du parcours. Depuis quelques années, la bataille gronde autour de ces diagnostics. Elle prend notamment la forme de grèves qui 
mettent en cause l'impact des calculs des directions d'établissement sur le dimensionnement des effectifs des différents bureaux.

L'activité du facteur est soumise à une exigence productive particulière : écouler chaque jour tout le courrier du jour. Il faut des circonstances exceptionnelles (par exemple le blocage des routes par la neige) pour qu'une direction d'établissement exonère les facteurs de cette obligation. En temps normal, il ne leur est permis de revenir au centre courrier que lorsque leur sacoche est vide. Dans ces conditions, si les combinaisons et les calculs automatisés sont erronés, inadéquats au regard des conditions effectives de l'activité, et plus particulièrement si les «durées théoriques » sous-estiment le temps nécessaire à la distribution de l'ensemble du courrier sur la tournée, le coût de l'écart à la réalité est assumé par le facteur, et non l'entreprise.

Le facteur assume ce coût par l'allongement de sa journée. Au-delà de la dégradation des conditions de travail que cela implique, il s'agit d'une perte sèche : les heures supplémentaires ne sont ordinairement pas enregistrées, donc pas rémunérées, car elles sont jugées irrecevables. Pour les directions, les heures supplémentaires ne contredisent pas le calcul préalable de la durée de la tournée, elles sont au contraire contredites par lui. Confrontés à l'étalon des «durées théoriques », les dépassements horaires ne peuvent signifier que la déviance, la paresse ou l'incompétence du facteur, et sont à ce titre considérés comme illégitimes.

Le seul moyen pour le facteur de faire à nouveau correspondre la durée reconnue par La Poste avec la durée effective de son travail, c'est d'accélérer son rythme, intensifier son activité. Cela passe par des prises de risque (par une vitesse excessive des déplacements) ou une dégradation de la qualité de service (par des modes transgressifs d'écoulement du trafic, dont témoignent à l'extrême les destructions de courrier parfois rapportés par la presse locale sous la forme de faits divers).

L'enjeu, le nœud de la controverse (qui a conduit jusqu'à la mise en examen de La Poste pour " travail dissimulé ») renvoie donc à cette question : les modélisations de l'entreprise sous-estiment-elles les durées réelles ? On ne peut répondre à cette question qu'en re-décomposant les éléments du modèle. D’abord, la schématisation de l'environnement géographique des tournées ne laisse filtrer qu'une partie des particularités locales, et même celles qui font l'objet d'un procédé de collecte ne sont pas toujours relevées systématiquement, par manque de temps des facteurs qui en sont chargés. Ensuite, l'évaluation du trafic est l'objet d'approximations, faute notamment d'un calibrage adéquat ou rénové des petits colis issus du e-commerce et des « nouveaux services » que doivent réaliser les facteurs (« veiller sur mes parents », relevés de compteurs, « remise commentée » de catalogues, administration de questionnaires, etc.). Enfin, il y a les «normes et cadences », 


\section{Les horaires des facteurs}

Ce que la Poste appelle « durée hebdomadaire de travail » (DHT) correspond en réalité à la durée hebdomadaire pendant laquelle un facteur, qu'il soit titulaire de la tournée ou remplaçant, est affecté à la réalisation du travail impliqué par une tournée qui doit desservir les usagers du lundi au samedi. C'est pourquoi cette DHT varie de 35 heures à 42 heures, et parfois au-delà. Bien souvent, la durée fixée par l'organisation à la journée de travail des facteurs est inférieure à 7 heures (c'est-à-dire que la DHT est inférieure à 42 heures), et les facteurs travaillent six jours sur sept. Lorsque la durée journalière programmée est supérieure à 5 heures 50 (c'est-à-dire que la DHT est supérieure à 35 heures), les facteurs bénéficient en contrepartie de repos de cycle. Lorsque la DHT est de 42 heures, c'est-à-dire que la journée de travail est de 7 heures, alors les facteurs bénéficient généralement d'un jour de repos glissant chaque semaine.

L'horaire des prises de service varie d'un centre à l'autre, et parfois d'une équipe à l'autre au sein d'un même centre. Elle dépend de choix stratégiques des directions d'établissement, parfois de compromis avec les facteurs, mais aussi de contraintes d'acheminement (un bureau situé en altitude et accessible d'un seul côté recevra son courrier en dernier, obligeant les facteurs à une prise de service tardive).

Le régime historique du temps de travail chez les facteurs est celle du service continu, sans pause, ou du moins avec une courte pause considérée comme du temps de travail. Aussi la durée légale du travail se confond-elle avec l'amplitude entre la prise et la fin de service (Barrois, Devetter, 2017). Mais, depuis quelques années, La Poste cherche à généraliser l'instauration d'une " pause méridienne " de 45 minutes, non comptée comme du temps de travail, qui allonge non seulement la durée effective du travail (car l'ancienne pause décomptée comme du temps de travail est supprimée) mais l'emprise du travail dans la journée du facteur, puisque l'écart entre la prise et la fin de service s'accroît.

personnage principal du présent article. La Poste les a conçues il y a plus de vingt ans dans des conditions qu'elle ne veut ou ne peut plus expliciter et justifier, mais elles sont supposées fonctionner aujourd'hui comme hier, en montagne comme en plaine, et sous tous les climats.

\section{III.3. L'évanouissement du référent}

Depuis quelques années, la contestation des facteurs et de leurs représentants ne porte plus sur le seul produit des calculs automatisés, c'est-àdire les «durées théoriques » justifiant des suppressions d'emploi et des rallongements de tournée. La contestation prend aussi de plus en plus la forme d'une interpellation sur les fondements mêmes des calculs, par le 
biais d'une institution de représentation du personnel nouvelle à La Poste : le comité d'hygiène, de sécurité et des conditions de travail (CHSCT).

Les cadences mises en œuvre à La Poste ont été une cible toute particulière des CHSCT et des experts qu'ils ont mandatés. Depuis 2015, cela a même conduit des tribunaux à ordonner à La Poste de livrer l'ensemble des documents justifiant ses cadences, voire à lui interdire d'entreprendre des suppressions de tournées avant d'avoir produit ces documents. Or, de telles décisions de justice débouchent sur une impasse car, en dehors de quelques documents épars et incomplets, La Poste affirme avoir tout perdu. Concernant la distribution, il n'y a rien de plus détaillé que l'étude de 1994 citée plus haut, qui dessine à grands traits les modalités de production des cadences, mais ne reproduit ni les résultats des chronométrages, ni les pondérations et les formules qui ont permis de déterminer des cadences univoques à partir d'observations nécessairement disparates. Selon La Poste, il ne serait pas possible d'en dire plus que ce qu'on a décrit dans cet article, c'est-à-dire pas assez pour jauger la rigueur d'un protocole et déterminer la fiabilité de ses résultats. «Le CHSCT rappelle notamment qu'une grande partie des données brutes ayant conduit à l'élaboration des

\section{Encadré 3}

\section{Les CHSCT à La Poste}

Le mouvement de " privatisation » de La Poste (au sens où le statut juridique de l'entreprise et de ses salariés quitte progressivement le droit public et bascule dans le droit privé) a eu notamment pour conséquence l'institution de CHSCT de plein exercice à partir de 2011. Dès la réforme de 1990, il est prévu que la partie du Code du travail traitant des CHSCT s'applique aux postiers. Mais c'est seulement après la transformation de La Poste en société anonyme (en 2010) que les CHSCT de La Poste ont obtenu toutes les prérogatives du privé, ne gardant de la fonction publique que les règles de désignation de ses membres (par les organisations syndicales et non, comme dans le privé, par les autres élus du personnel). Le basculement des instances de représentation du personnel est demeuré partiel, puisque les agents de La Poste n'ont ni délégués du personnel ni comités d'établissement et d'entreprise (ce qui pose dans des termes particuliers la question du basculement, aujourd'hui, vers le nouveau « comité social et économique »). L'avis du CHSCT doit être sollicité lors de chaque réorganisation, suppression de tournées, introduction de nouveaux outils ou dispositifs organisationnels. Le CHSCT peut à cette occasion solliciter l'appui d'un expert extérieur. Les représentants du personnel au sein de cette instance n'ont qu'un avis consultatif, mais ils peuvent tenter de bloquer en justice la mise en œuvre d'une réorganisation au nom du manque d'information ou de l'impact délétère que la nouvelle organisation aurait sur les conditions de travail. 
études n'est pas communiquée, plaide ainsi un avocat de la direction dans une de ces affaires. La Poste ne prétend pas le contraire ! En revanche, force est de constater que ces éléments n'existent pas ${ }^{33}$ !»

Le contraste est saisissant. Dans les centres courrier, les directions locales opposent aux facteurs sceptiques le caractère scientifique des calculs. Or, derrière la masse opaque, complexe et impressionnante que charrie le mot " science ", il s'avère que les directions masquent l'évanouissement du référent originel. À en croire la défense de la direction nationale, nul ne peut aujourd'hui consulter le registre des expériences à l'origine des cadences, et nul ne peut plus en vérifier la robustesse. À présent que des facteurs remontent la longue chaîne des opérations et des équivalences qui gouvernent leurs tournées, c'est pour s'apercevoir qu'elle n'est amarrée nulle part.

\section{Conclusion}

Le présent article participe de ce qu'il raconte, puisqu'il cherche à rendre raison des cadences utilisées à La Poste en rappelant l'arbitraire de leurs origines. Il ne se contente donc pas de restituer une controverse, il la nourrit en contredisant le discours de légitimation qu'utilisent les directions de La Poste, qui associe oubli de la genèse et prétention scientifique.

Les temps standards constituent un "investissement de forme » (Thévenot, 1986 ; Ghaffari et al., 2013) qui accroît la maitrise et la prévisibilité de l'organisation de la production pour ceux qui ont le mandat de (et espèrent réussir à) diriger. Leur conception, et plus encore leurs usages, ont suivi un cheminement heurté. La Poste a mis environ 20 ans à concevoir des « temps virtuels » et des « durées théoriques » de la distribution du courrier, et il a fallu encore près de dix années pour qu'elle les substitue aux classiques chronométrages.

En calculant des cadences, au creux de l'hiver 1994, La Poste avait-elle l'intention de s'en servir pour prescrire le travail des facteurs? Ce n'est pas sûr. Le changement d'usage est peut-être le fruit de rationalisations ultérieures et contingentes. On peut faire l'hypothèse que la réduction de la durée légale du travail et la baisse du trafic, dans un contexte de libéralisation du secteur, ont conduit La Poste à réviser rapidement sa position et, pour gagner du temps, à puiser dans les vitesses et durées conventionnelles déjà disponibles. Cela expliquerait la négligence qui l'amène aujourd'hui à avouer avoir perdu les fondements des outils qui gouvernent la vie au travail de 70000 de ses subordonnés.

33. «Conclusions en réponse » de la société La Poste, TGI de Paris, audience du 9 mai 2017, $n^{\circ} R G$ : $17 / 51830$. Source syndicale. 
À présent, le roi est-il nu, puisqu'il concède que ses constructions rationalisatrices ont perdu leurs fondations? On pourrait le croire, en lisant que La Poste s'engage par accord, début 2017, à ouvrir " un chantier portant sur l'ensemble de l'évolution des normes et cadences à la distribution ${ }^{34} »$. Mais, dix ans plus tôt, elle avait déjà convenu d'ouvrir " une concertation nationale sur les normes et cadences ${ }^{35}$ ", sans que cela conduise ne serait-ce qu'à des amendements des principaux temps standards. Pourtant, à intervalles réguliers, La Poste continue d'échantillonner des tournées et de chronométrer des facteurs ${ }^{36}$. Dans une mesure qui demeure inconnue, car cachée, l'entreprise actualise sa propre information (ainsi que celle de l'autorité de régulation postale), mais ne corrige pas les paramètres qui organisent la production et déterminent les conditions de travail des facteurs.

C'est que rouvrir cette « boîte noire » (Callon, Latour, 2006) risquerait de mener loin. Nous ne pouvons ici que suggérer des pistes. Il faudrait d'abord réactualiser des durées, rediscuter des paramètres qu'elles intègrent, ou qu'elles oublient (comme le vieillissement des facteurs), enregistrer le changement d'environnement qui les détermine (comme la densification de la circulation). Ensuite, il faudrait questionner la fabrication de durées théoriques pensées comme des moyennes, autour desquelles la durée du travail quotidienne du facteur fluctuerait, car cette variation incessante des horaires effectifs, commandée par une préquantification du travail, n'est pas juridiquement compatible avec le statut de salarié qui, plus de 15 ans après la fin des concours de fonctionnaires, est désormais majoritaire parmi les facteurs.

Enfin, l'enjeu est d'autant plus important, et l'issue d'autant plus incertaine, qu'il ne concerne pas uniquement La Poste mais l'ensemble du secteur postal. Si le roi n'est pas nu, c'est aussi parce qu'il est tenu : les conventions de La Poste sont devenues celles de l'autorité de régulation du secteur, qui fonde sur elles les règles qu'elle édicte pour organiser la concurrence. Les temps virtuels imposés aux facteurs sont (re-)traduits en coûts comptables sur lesquels est fondée la réglementation de la tarification des plis et colis. De proche en proche, partie de la tournée des facteurs pour remonter aux conventions les plus agrégées et enchevêtrées, la remise en cause pourrait ébranler jusqu'aux investissements de forme qui, au cours des deux dernières décennies, ont été nécessaires à la constitution volontariste et laborieuse d'un « marché » postal.

34. Article 2 de l'Accord sur l'amélioration des conditions de travail et sur l'évolution des métiers de la distribution et des services des factrices/facteurs et de leurs encadrantes/encadrants de proximité, 7 février 2017 (signé par la CFDT, FO et la CFTC).

35. Engagement $n^{\circ} 7$ de l'article 2 de l'accord relatif au développement professionnel des facteurs, 19 janvier 2007.

36. On en a une trace dans une étude d'économistes de La Poste dont certains éléments ont été rendus publics (Bernard et al., 2010), qui (ré-)évalue le temps nécessaire au tri et à la distribution des « objets ordinaires " (ni suivis ni recommandés) selon leur poids et leur format. Par rapport aux normes et cadences de 1994, cette étude introduit de nouvelles distinctions entre catégories d'objets, auxquelles elle impute des durées différentes, qui n'ont cependant pas de traduction dans la prescription de la charge de travail des facteurs. 


\section{Références bibliographiques}

Adam H. (2010), « Privatisation réalisée et privatisation en cours : des télécommunications à La Poste », in Pelletier W., Bonelli L. (dir.), L'État démantelé, Paris, La Découverte, p. 260-265, https://www.cairn.info/l-etat-demantele-9782707160195-p-260.htm.

Barrois A., Devetter F.-X. (2017), « Durées courtes, journées longues. Fragmentation du temps de travail et pression sur le salaire ", La nouvelle revue du travail, $\mathrm{n}^{\circ} 11$, https://doi.org/10.4000/nrt.3272.

Bernard S., Gomez C., Martin L., Roy B. (2010), « Determining the Impact of Shape and Weight of Mail Items on Manual Processing Costs: An Experimental Approach », in Crew M.A., Kleindorfer P.R. (eds.), Heightening Competition in the Postal and Delivery Sector, London, Edward Elgar, p. 338-354.

Bonnet P. (2015), « Pour une histoire sociale de l'analyse des données », in Lebaron F. (dir.), La méthodologie de Pierre Bourdieu en action, Paris, Dunod, p. 21-42, https://doi.org/10.3917/dunod.lebar.2015.01.0021.

Bras B. (1995), La Poste entre le service public et le marché, Thèse de doctorat en économie des institutions, Université Paris X.

Callon M., Latour B. (2006), « Le grand Léviathan s'apprivoise-t-il ? » in Akrich M., Callon M., Latour B. (dir.), Sociologie de la traduction. Textes fondateurs, Paris, Presses des Mines, p. 11-32.

Cartier M. (2003), Les facteurs et leurs tournées. Un service public au quotidien, Paris, La Découverte, https://www.cairn.info/les-facteurs-et-leurs-tournees--9782707139832.htm.

Cazals C., de Rycke M., Florens J. P., Rouzaud S. (1997), « Scale Economies and Natural Monopoly in the Postal Delivery: Comparison Between Parametric and Non Parametric Specifications ", in Crew M.A., Kleindorfer P. R. (eds.), Managing Change in the Postal and Delivery Industries, Boston, Springer, p. 65-80, https://doi.org/10.1007/978-1-4615-6321-1_4.

Cazals C., Fève F., Florens J.-P., Roy B. (2005), « Delivery Costs II: Back to Parametric Models ", in Crew M.A., Kleindorfer P.R. (eds.), Regulatory and Economics Challenges in the Postal and Delivery Sector, Boston, Kluwer, p. 189-202.

Chatzis K. (1999), « Searching for Standards: French Engineers and Time and Motion Studies of Industrial Operations in the 1950s », History and Technology, vol. 15, n³, p. 233-261, https://doi.org/10.1080/07341519908581948.

Cour des comptes (2016a), « Les facteurs face au défi de la baisse du courrier : des mutations à accélérer », in Rapport public annuel 2016, p. 397-428, https:// goo.gl/YbTDnk.

Cour des comptes (2016b), La Poste : une transformation à accélérer, https://www. ccomptes.fr/fr/publications/la-poste-une-transformation-accelerer.

Demazière D. (2005), « Au cœur du métier de facteur : "sa" tournée », Ethnologie française, vol. 35, n 1, p. 129-136, https://doi.org/10.3917/ethn.051.0129.

Demazière D., Mercier D. (2003), « La tournée des facteurs. Normes gestionnaires, régulation collective et stratégies d'activité ", Sociologie du Travail, vol. 45, $\mathrm{n}^{\circ} 2$, p. 237-258, https://doi.org/10.1016/S0038-0296(03)00016-5. 
Devetter F.-X., Rousseau S. (2003,), « Travail relationnel et contrôle hiérarchique : pourquoi La Poste modifie-t-elle les circuits de distribution des facteurs ? ", IX Journées de sociologie du travail, 27-28 novembre, Paris.

Gadrey J. (2000), La productivité du travail à La Poste (1970-1998), Mission recherche de La Poste.

Ghaffari S., Misset S., Pavis F., Ponnet M. (2013), « Généalogies des outils de gestion. Introduction », Travail et emploi, $n^{\circ} 133$, p. 5-12, https://www.cairn.info/ revue-travail-et-emploi-2013-1-page-5.htm.

Hatzfeld N. (2005), « Du règne du chronomètre au sacre du temps virtuel. Une histoire de succession aux usines Peugeot (1946-1996) », in Linhart D., Moutet A. (dir.), Le travail nous est compté, Paris, La Découverte, p. 60-70, https://www.cairn.info/le-travail-nous-est-compte--9782707144645-p-60. $\mathrm{htm}$.

La Poste (2002), Voyage au centre du courrier. La production courrier en dix chapitres, Paris, Direction du courrier.

Maynard H.B., Stegemerten G.J., Schwab J. (1953), MTM. Méthodes de travail et tables de temps, Paris, Gauthier-Villars.

Roy B. (1999), "Technico-Economic Analysis of the Costs of outside Work in Postal Delivery », in Crew M.A., Kleindorfer P.R. (eds.), Emerging Competition in Postal and Delivery Services, Boston, Springer, p. 101-122, https:// doi.org/10.1007/978-1-4757-5122-2_8.

Salaün M. (2008), Le métier de facteur à l'épreuve des nouvelles organisations du travail à La Poste, Thèse de doctorat en sociologie, Université Paris-Est École nationale des Ponts et Chaussées, https://pastel.archives-ouvertes.fr/ tel-00481835/document.

Samzun T. (2007), La fin d'un patronage d'État. Consentement et conflit au travail. Le cas des facteurs de la Poste dans les Bouches-du-Rhône, Thèse de doctorat en sociologie, Université Aix-Marseille I, https://tel.archives-ouvertes. fr/tel-00198931/document.

Thévenot L. (1986), « Les investissements de forme », in Thévenot L. (dir.), Conventions économiques, Paris, CEE-Puf, p. 21-71. 